\title{
Erratum to: Numerical Representability of Ordered Topological Spaces with Compatible Algebraic Structure
}

\author{
Juan C. Candeal · Esteban Induráin • José A. Molina
}

Published online: 11 November 2011

(C) Springer Science+Business Media B.V. 2011

\section{Erratum to: Order (2011) \\ DOI 10.1007/s11083-011-9202-8}

Unfortunately, we found a significant omission in the article. In Definition 3.5 a condition was missing. That condition is important because it has a decisive influence on the proof of the main Theorem 3.14, as well as on the footnote linked to Definition 3.5.

Without the condition to be added in Definition 3.5, the proof of Theorem 3.14 has a flaw. The reason is that the quotient $X / I(\mathbf{0})$ could be the zero-ring: multiplying any two given classes in $X / I(\mathbf{0})$ the product could be always zero.

Adding the new condition to Definition 3.5 that situation is avoided, and the given proof of Theorem 3.14 is entirely valid.

The corrected version of Definition 3.5 reads as follows. Its corresponding footnote has been here changed accordingly.

Definition 3.5 Let $X$ be a real algebra. A total preorder $\precsim$ defined on $X$ is said to be multiplicative ${ }^{1}$ if

(i) $x \precsim y$ implies $(z * x \precsim z * y, x * z \precsim y * z),(x, y, z \in X, \mathbf{0} \precsim \mathrm{z})$,

(ii) $x \prec y$ implies $(z * x \prec z * y, x * z \prec y * z),(x, y, z \in X, \mathbf{0} \prec \mathrm{z})$.

\footnotetext{
${ }^{1}$ If $*$ is zero, then the only total preorder that is multiplicative is the trivial one (i.e., $x \sim y$, for all $x, y \in X)$.

The online version of the original article can be found at http://dx.doi.org/10.1007/s11083-011-9202-8.

J. C. Candeal · J. A. Molina

Departamento de Análisis Económico, Facultad de Ciencias Económicas y Empresariales, Universidad de Zaragoza, Zaragoza, Spain

E. Induráin $(\bowtie)$

Departamento de Matemáticas, Universidad Pública de Navarra, Campus Arrosadía,

Edificio las Encinas, 31006 Pamplona, Spain

e-mail: steiner@unavarra.es
} 